\title{
La migración en la reproducción de la sociedad global
}

\author{
ALEJANDRO I. CANALES*
}

RESUMEN: En este texto presentamos una propuesta teórico-metodológica alternativa con la que queremos contribuir al diseño de un marco comprehensivo de la migración en la sociedad contemporánea. Nuestra tesis es que en la actualidad, la migración internacional es un fenómeno que desde diversos ámbitos y desde diversos niveles, contribuye a la estructuración de la sociedad contemporánea. Para entender este proceso de estructuración, proponemos analizar la migración como un factor de la reproducción de la sociedad, y por ese medio, de su transformación. Sin duda, el modelo de la reproducción configura un gran rompecabezas con infinidad de piezas, muchas de las cuales además, se superponen y engarzan de más de una manera. En nuestro caso, hemos optado por analizar sólo tres de ellas, y que corresponden a la migración como factor de reproducción de las poblaciones, de reproducción del capital, y de reproducción de las estructuras de clases y de desigualdad social. Con este modelo, queremos avanzar en el entendimiento no sólo de la migración, sino que además, y a través de ella, en un mejor entendimiento de la sociedad contemporánea. En tal sentido, identificamos la posición, función, y contradicciones que la migración plantea en cada uno de estos tres grandes momentos de la reproducción de la sociedad: la demografía, la economía, y la estructura de clases sociales.

PALABRAS CLAVE: teoría de la migración, reproducción social, reproducción de la población, reproducción del capital, globalización.

ABSTRACT: In this paper we present a theoretical and methodological model for the analysis and understanding of migration in contemporary society. Our thesis is that at present, the international migration phenomenon from different areas and from different levels, contributes to the structuring of contemporary society. To understand this process, we propose to analyze migration as a factor in the reproduction of society, and thereby, their transformation. Certainly, the reproduction model sets a great puzzle with many pieces, many of which also overlap and enshrine in more than one way. In our case, we discuss only three of them, corresponding to migration as a factor in population reproduction, reproduction of capital, and reproduction of class structures and social inequality. With this model, we advance the understanding not only of migration, but also, and through it, in a better understanding of contemporary society itself. In this sense, we identify the position, function, and contradictions that migration presents in each of these three great moments of the reproduction of society: demography, economics, and the social class structure.

KEY WORDS: theory of migration, social reproduction, reproduction of population, reproduction of capital, globalization.

* Docente-investigador del Centro Universitario de Ciencias Económico Administrativas (CUCEA) de la Universidad de Guadalajara, México. 


\section{INTRODUCCIÓN}

71 debate sobre migración y desarrollo está atravesado por una serie de sesgos político-ideológicos e inconsistencias teórico-metodológicas. La mayor debilidad que identificamos corresponde al nacionalismo metodológico que subyace en ellas, y que deriva en distorsiones y sesgos en el análisis sobre las causas, condiciones y consecuencias de la migración internacional. Se trata de una herencia epistemológica de la cosmovisión de la modernidad, con base en la cual todo el pensamiento moderno y la teoría social se habrían sustentado en el principio de correspondencia entre Estado, nación y territorio (Beck, 1998). Según este principio, el Estado nacional se circunscribe a sus confines territoriales y conforma una entidad de análisis, observación y teorización en sí misma, independiente de los otros Estados nación. A partir de ello, las sociedades nacionales se ven como un todo en sí mismas, conforman el contexto para el análisis de cualquier proceso social, y devienen la unidad de análisis para el estudio de los procesos mundiales, los que se conceptualizan como procesos y relaciones internacionales, esto es, entre-naciones.

Con el advenimiento de la sociedad global y posmoderna, sin embargo, este nacionalismo metodológico queda desfasado, sin lograr aprehender las nuevas dinámicas y formas que asumen los procesos sociales. En un mundo donde las sociedades se globalizan, en donde los procesos sociales, económicos, culturales y de todo tipo traspasan y disuelven cotidianamente las fronteras nacionales, resulta anacrónico seguir suponiendo que el Estado nación es la forma social natural del mundo contemporáneo (Wimmer y Glick Shiller, 2002). Tal parece ser el desafío en el caso de la migración internacional. En un mundo globalizado, basado en modelos económicos y patrones de acumulación globales, no cabe duda que la migración ha de ser pensada y analizada en términos de su globalización. Considerando esta visión crítica al nacionalismo metodológico, en este texto presentamos una propuesta teórico-metodológica, que contribuye al diseño de un marco comprehensivo de la migración en la sociedad contemporánea. 
Retomando a Giddens (1984), partimos de la premisa teórica de que la migración constituye un componente que contribuye a la estructuración de la sociedad global, y por tanto, a su dinámica y continuo devenir. Desde esta perspectiva, lo que resulta relevante, entonces, es analizar y debatir su papel en la dinámica y movimiento de la sociedad, esto es, sus contribuciones a la dinámica y transformación de la sociedad global. Para ello, proponemos analizar la migración desde el enfoque de la reproducción de la sociedad.

En las ciencias naturales, la reproducción representa la continuidad en el tiempo y en el espacio de determinadas estructuras, esto es, la permanencia de las condiciones materiales asociadas a la forma de existencia de tales estructuras. En tal sentido, Maturana (2009) plantea precisamente, que es el concepto de autopoiesis, esto es, auto-organización de su propia reproducción, lo que permite definir a los sistemas vivos.

En ciencias sociales, este concepto alude a la capacidad de la sociedad de auto-organización, incorporando las dinámicas, racionalidades, contradicciones y funcionalidades de los más diversos procesos, agentes y estructuras, y que le permiten y posibilitan su reproducción en el tiempo y el espacio. Sin embargo, la reproducción no puede confundirse ni reducirse a lo que sería un proceso de renovación pura y simplemente. Antes bien, la reproducción se refiere a un doble proceso de continuidad y cambio de las estructuras materiales y simbólicas, con lo cual se abre paso a un análisis conjunto de la formación, disolución y transformación de determinadas estructuras sociales. En tal sentido, el modelo de la reproducción exige una mirada hacia el movimiento de la sociedad, hacia su devenir y transformación continua. Asimismo, esta mirada del movimiento permite identificar los diversos factores que lo impulsan, a la vez que identificar las contradicciones y tensiones que se generan entre ellos.

Considerando lo anterior, el modelo de análisis propuesto centra su mirada en preguntarse por las formas en que la migración internacional contribuye y participa de la reproducción de la sociedad de nuestros tiempos. Con este modelo, queremos avanzar en el entendimiento no sólo de la migración, sino que además, y a través de ella, en un mejor entendimiento de la sociedad contemporánea. 
Sin duda, el análisis y problematización de la migración a partir de sus funciones, contribuciones y tensiones respecto a la reproducción de lo social, puede abordarse desde los más diversos campos sociales. En nuestro caso, hemos optado por delimitar el modelo de análisis a tres ámbitos sociales, a saber: a) la reproducción de la población (demografía); b) la reproducción del capital (economía), y c) la reproducción de la desigualdad social (estructura de clases).

Migración y reproducción demográfica

Vista desde la dinámica de la población mundial, la migración internacional contemporánea no sólo corresponde a una forma de desplazamiento de personas, sino que a través de ella se configura un sistema global de reproducción demográfica, que se sustenta en la complementariedad de la dinámica de población de las regiones de origen con la dinámica en los países de destino.

\section{Dinámica demográfica en países de destino}

En el caso de los países de destino (Estados Unidos y Europa, sobre todo), la migración permite llenar el vacío demográfico que están generando, simultáneamente, dos fenómenos demográficos complementarios, a saber: el descenso de la fecundidad en el marco de la llamada segunda transición demográfica, y el proceso de envejecimiento de la población en esos países como resultado del fin de la primera transición demográfica.

La segunda transición demográfica se trata de un modelo propuesto por Van de Kaa (1987) para explicar la dinámica demográfica en las sociedades europeas de fines del siglo xx que experimentaran un continuo descenso en los niveles de fecundidad, llegando en algunos casos a estar incluso por debajo de los niveles que asegurarían la reproducción demográfica. Este descenso se sustentaría en los procesos de individuación de la vida social y familiar, contribuyendo al debilitamiento de la familia como institución, y un reforzamiento del individuo, sus derechos y su autorrealización en diversos

$12 \frac{\text { SEGUNDO SEMESTRE } 2013}{\text { MIGRACIÓN Y DESARROLLO NÚM. } 21}$ 
planos de la vida social, económica, política y cultural, que trascienden los estrechos círculos familiares (Beck y Beck, 2002).

Las consecuencias demográficas de este fenómeno son de dos tipos. Por un lado, implica un cambio en la composición y dinámica de los hogares y familias, y por otro, un continuo descenso en los niveles de fecundidad. En el primer caso, el tradicional modelo de familia nuclear ha quedado desfasado, imponiéndose una variedad y diversidad de patrones de uniones y de familias, las cuales, además, presentan una evolución y cambio de gran dinamismo. Frente al descenso de las familias nucleares tradicionales, se antepone el incremento de las personas que viven solas, así como de familias monoparentales, familias recompuestas y de hogares compuestos.

En el segundo caso, los datos son elocuentes. En el caso de España, por ejemplo, entre 2000 y 2011, la tasa global de fecundidad de las mujeres nativas era apenas de 1.3 hijos por mujer, muy por debajo del nivel mínimo que asegura la reproducción de la población. En Estados Unidos, la situación no es muy distinta, allí la tasa global de fecundidad de las mujeres nativas no latinas es de 1.83 hijos por mujer. ${ }^{1}$ Estas excesivamente bajas tasas de fecundidad y reproducción se deben ya no tanto al impacto del uso de métodos modernos de anticoncepción (los cuales ya son de uso generalizado por la población) como a factores sociales que han modificado el comportamiento y actitud frente a los hijos y la descendencia, y que se manifiestan en un mayor retardo en la edad al tener el primer hijo, al aumento de madres con un solo hijo y al incremento de parejas y de mujeres que no desean tener hijos (Herrera, 2007).

El proceso de envejecimiento se refiere al cambio en la composición y estructura etárea de la población como resultado de los cambios en la dinámica de la mortalidad y de la fecundidad, que se consolidaron bajo el contexto de la primera transición demográfica. Se llama envejecimiento, pues la tradicional estructura por edades de la población, que adoptaba una forma piramidal, con una base amplia producto de las altas tasas de fecundidad y natalidad, y una cúspide baja y angosta, producto de los altos niveles de

${ }^{1}$ Cálculos propios con base en US Department of Health and Human Services (2012), para Estados Unidos, e INE (2013), para España. 
mortalidad, comienza a adquirir una forma más bien de una ojiva, con una base en continuo estrechamiento, derivado de la reducción de los nacimientos, y una cúspide que a la vez que se eleva, también se ensancha, producto de la reducción de la mortalidad, y el incremento en la esperanza de vida de las personas.

Estas tendencias configuran una peculiar situación demográfica, caracterizada por una desfavorable relación de dependencia demográfica que impacta negativamente en la capacidad de reproducción económica de la población, en la medida que tiende a reducirse la fuerza de trabajo, especialmente en edades jóvenes, menores de 50 años (Cooke, 2003). En el caso de Estados Unidos, por ejemplo, entre 2000 y 2010 la población de 15 a 49 años, que corresponde a la población en plenas edades productiva y reproductiva, se incrementó en casi 5.5 millones de personas. Sin embargo, mientras la población nativa no hispana en estas edades se redujo en 3.5 millones de personas, la población de origen hispano se incrementó en 7.7 millones de personas. Asimismo, en el caso de España, la situación es prácticamente la misma. Entre 2000 y 2010, la población de 15 a 49 años se incrementó en casi 3.7 millones de personas. Sin embargo, mientras los nacidos en España se redujeron en más de 1.1 millón de personas, los inmigrantes internacionales en esas edades se incrementó en 3.8 millones de personas.

Estos datos ilustran cómo en Estados Unidos, España y, de forma similar, en otros países desarrollados de Europa, la inmigración desde países periféricos contribuye no sólo a llenar el vacío demográfico que deja la dinámica de sus poblaciones, sino que además estaría conformando una base para el virtual reemplazo demográfico.

Es el caso de Estados Unidos, por ejemplo, si hacia 1970 era innegable la supremacía de los blancos no latinos, quienes representaban $83 \%$ del total de la población estadounidense. Hacia 2010, en cambio, esta supremacía se habría reducido, de modo de que este grupo étnico aportaba menos de 65\% de la población. Asimismo, de continuar con estas tendencias, se estima que para 2050, los blancos no latinos representen sólo 46.3\% de la población, dejando de ser, por tanto, una mayoría absoluta.

$14 \frac{\text { SEGUNDO SEMESTRE } 2013}{\text { MIGRACIÓN Y DESARROLLO NÚM. } 21}$ 
Por el contrario, los altos volúmenes de inmigración mexicana y latinoamericana, junto a su elevada fecundidad, plantean el escenario inverso. Si en 1970 representaban menos de 5\% de la población total, ya para 2010 alcanzaban a ser $16 \%$ de la población estadounidense, y se proyecta que para 2050 este grupo étnico represente algo más de 30\% de la población. Esta dinámica de crecimiento llevaría a que este grupo étnico deje de ser una simple minoría étnica, para convertirse en un grupo con la fuerza demográfica suficiente que la ubicaría como la segunda mayoría relativa, cuestionando directamente la tradicional primacía demográfica de la población blanca no latina. Estas diferentes y opuestas dinámicas demográficas, derivarían en un proceso de reemplazo étnico y demográfico, el cual ya se manifiesta en el caso de la población de algunos estados como California y Texas, así como en las ciudades de Los Ángeles, Miami y Houston, en donde el volumen de la población de origen latino ya es igual o superior al de la población blanca no latina (Canales, 2011a).

\section{Dinámica demográfica en países de origen}

En el caso de los países de origen, especialmente en América Latina, asistimos, en cambio, a la última fase de su transición demográfica, previa al envejecimiento de su población (Guzmán, 2002). En esta fase se da una reducción del volumen absoluto y relativo de la población infantil, así como un incremento también en términos absolutos y relativos de la población en edades activas. Asimismo, la población adulta mayor, aún cuando crece en términos absolutos, se mantiene en bajos montos demográficos. Esta combinación de tendencias demográficas provoca una coyuntura histórica única. Desde hace algunos lustros y en las próximas décadas la carga que representa la población inactiva será muy inferior con relación a la de otras coyunturas históricas. Es por ello que a esta peculiar situación se la ha denominado bono demográfico, ya que se quiere enfatizar la favorable situación en términos de relaciones de dependencia y carga económica, que implica esta reducción de la población en edades inactivas (Redondo y Garay, 2012). En este contexto, la migración internacional configura una forma de exportación 
de este bono demográfico hacia los países del Primer Mundo, los cuales pasan también por una coyuntura especial de déficit de población en edades jóvenes y activas.

En América Latina la población en edad activa (15 a 64 años) se incrementó en casi 65 millones de personas, entre 2000 y 2010. De ellos, sin embargo, más de 10\% habrían emigrado a Estados Unidos o España (principales destinos de la emigración latinoamericana). Pero este es un promedio regional, la situación es aún más grave en el caso de aquellos países de mayor emigración. En el caso de México, por ejemplo, se estima que la pérdida de este "bono demográfico» haya alcanzado a casi $22 \%$ en esa década. Similar situación se daría en Guatemala, Ecuador, Honduras y República Dominicana. Los casos extremos son El Salvador y Cuba, en donde se estima que 42\% y $36 \%$ de su «bono demográfico», respectivamente, haya emigrado a Estados Unidos y en menor medida a España.

\section{Hacia un régimen global de reproducción demográfica}

Nuestra hipótesis es que, a través de la migración internacional, se configura un modelo de reproducción de la población que articula estas diferentes dinámicas de la población. Si bien se trata de dinámicas demográficas localmente situadas, mediante la migración internacional adquieren un sentido y significado global que trasciende sus fronteras nacionales.

La migración internacional contribuye así a la configuración de un modelo de reproducción demográfica propio de esta era de globalización. En efecto, a través de la migración se fomenta la transferencia de parte de este bono demográfico desde los países periféricos, latinoamericanos en particular, lo cual contribuye, a su vez, a solventar el déficit de población en edades activas que genera el envejecimiento de la población en los países desarrollados. Se trata, en definitiva, de la configuración, a nivel global, de un sistema de complementariedad demográfica, que permite dar sustento a la reproducción de la población en los países de destino y de origen. En el esquema 1, ilustramos este modelo de complementariedad demográfica, con sus respectivas tensiones y posibles consecuencias.

$16 \frac{\text { SEGUNDO SEMESTRE } 2013}{\text { MIGRACIÓN Y DESARROLLO NÚM. } 21}$ 
ESQUEMA 1

Migración internacional y reproducción de la población

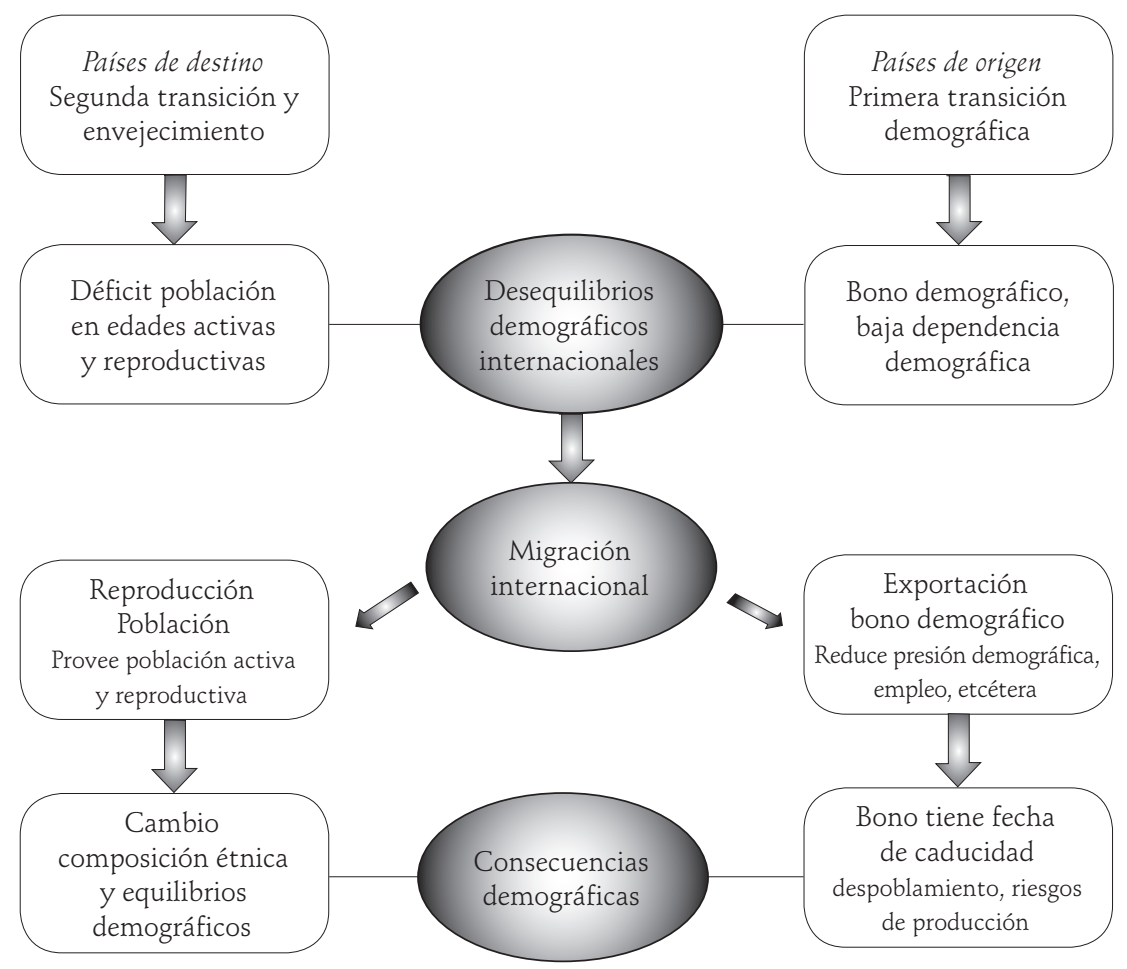

Fuente: Elaboración propia.

Sin embargo, este sistema global de reproducción demográfica no está exento de tensiones y contradicciones. Por un lado, la consecuencia en los países de destino es que la masividad de la inmigración puede derivar en un virtual reemplazo demográfico de población nativa por inmigrantes, transformando con ello la composición étnica y migratoria de la población (onU, 2001). Cabe señalar, sin embargo, que la masividad de la inmigración no es sino la otra cara de la magnitud del déficit demográfico en los países de destino.

Por su parte, en los países de origen, si bien la masividad de la emigración de población a corto plazo reduce la presión que genera el bono demográfico, a mediano y largo plazo el efecto puede ser contraproducente. La reducción de la población en edades reproductivas deriva a mediano plazo en una re- 
ducción de la natalidad, a lo cual si le agregamos la reducción de la fecundidad que ya se experimenta en estos países, entonces estaríamos en un escenario en que se compromete la capacidad de reproducción demográfica. Tal es el caso de Cuba, por ejemplo, que es uno de los países de la región que más ha avanzado en la transición demográfica y donde la tasa global de fecundidad desde hace ya un par de décadas está por debajo del nivel mínimo para garantizar la reproducción demográfica de la población.

\section{Migración y reproducción del capital}

Desde la teoría económica, el funcionamiento de la economía se analiza como un sistema de circulación que va en dos sentidos. Por un lado, la circulación o flujo de lo material (bienes y servicios) y, por el otro, el flujo o circulación del dinero (ingresos, rentas, etcétera). A este modelo se le llama, precisamente, flujo circular de la renta o también flujo circular de la actividad macroeconómica. La mirada conjunta de los dos flujos permite entender el funcionamiento de la economía. Veamos esto con base en el modelo más sencillo, pero por lo mismo, más esclarecedor, y que corresponde al intercambio económico entre sólo dos agentes: las unidades domésticas, por un lado, y las unidades productivas, o empresas, por el otro.

Las unidades domésticas son familias o personas que participan de dos modos en el sistema económico. Por un lado, son los propietarios de los factores de producción (capital, tierra y fuerza de trabajo) y, por otro lado, demandan bienes y servicios para satisfacer sus múltiples necesidades. Por su parte, las empresas son unidades donde, con estos factores productivos se producen los bienes y servicios que se ofrecen a las familias para su subsistencia y manutención. ${ }^{2}$

${ }^{2}$ Este modelo se puede complejizar agregando nuevos agentes, mercados y componentes del sistema económico, tales como el Estado y las empresas y consumidores del exterior, los intercambios entre empresas, la producción y circulación de insumos, bienes de capital, tecnología, y todo ello diferenciando además según los distintos sectores de producción. Para más detalles, véase Castro y Lessa (1991).

$18 \frac{\text { SEGUNDO SEMESTRE } 2013}{\text { MIGRACIÓN Y DESARROLLO NÚM. } 21}$ 
Si bien desde la teoría económica neoclásica el modelo es usado para analizar el flujo de la renta, y por ese medio, las condiciones para el crecimiento de la actividad económica, así como su distribución entre los diferentes agentes económicos, desde una perspectiva estructuralista podemos usar el mismo modelo de funcionamiento del sistema económico, pero para vincular los procesos de consumo y producción. Entendiendo el primero como el proceso de reproducción de la fuerza de trabajo y el segundo como el proceso de reproducción del capital, y siguiendo el mismo esquema del flujo circular de la renta, podemos entender el funcionamiento de la economía como la complementación de dos momentos diferentes, a saber:

Momento productivo: corresponde a la producción tanto de los medios de producción y medios de consumo intermedio (maquinarias, insumos, materias primas, tecnología, etcétera), así como a la producción de bienes y servicios de consumo final. En este proceso, el capital consume la fuerza de trabajo, es decir, extrae de ella lo que tiene valor para el capital: el trabajo, que no es sino la capacidad del trabajador para usar los demás medios de producción, insumos y demás en la producción de bienes y servicios que permitan valorizar el capital invertido inicialmente.

Momento reproductivo: corresponde al proceso de consumo individual (y familiar) de los bienes y servicios producidos previamente. A través de este proceso las personas no sólo satisfacen sus necesidades (perspectiva neoclásica), sino que se reproducen a sí mismas, y por ese medio reproducen cotidianamente su propia fuerza de trabajo (perspectiva estructuralista). Si para los individuos este momento de consumo individual no representa un acto productivo propiamente, esto es, que les reditúe algo más que la satisfacción de sus necesidades, para el capital, en cambio, constituye un momento productivo, pues corresponde a la producción y reproducción de la fuerza de trabajo.

Este es un modelo general que describe el funcionamiento de la economía capitalista en abstracto. Lo que nos interesa en esta ocasión es reconstruir este modelo para el análisis y entendimiento del papel de la migración contemporánea en el proceso de reproducción del capital en el actual contexto de globalización económica. 
Distintos autores se han referido con detalle a los procesos de globalización de la economía, poniendo particular énfasis en cómo habría influido en las modalidades y patrones de la migración internacional contemporánea (Sassen, 2007; Castles y Miller, 1993). En particular, nos interesa ilustrar cómo en este contexto de globalización la migración internacional constituye un mecanismo que permite la vinculación de los momentos productivos (de valorización y reproducción del capital) con los momentos reproductivos de la fuerza de trabajo.

En relación con el llamado momento productivo, diversos autores señalan que estaríamos transitando desde el ya clásico modelo industrial de producción en masa de tipo fordista, a uno de producción flexible, just in time, basado en los principios de organización industrial postfordistas (Lipietz, 1997). Si en el primero se articulaba una base productiva nacional con una comercialización mundial, en el segundo la globalización ha alcanzado también al mismo proceso de trabajo. Asimismo, si el primero daba origen a un orden social y estatal, identificado con el keynesianismo y el Estado de bienestar como marcos de regulación de la economía, el segundo da origen a un nuevo orden económico y social mundial sustentado en el neoliberalismo y el mercado como mecanismo de (des)regulación del funcionamiento de la economía. De esta forma, con la globalización, la producción flexible y la desregulación especialmente en el mundo laboral, se ha dado origen a un régimen de riesgo laboral (Beck, 2000) que reemplaza al antiguo régimen laboral que surgiera al amparo del Estado de bienestar, especialmente en los países desarrollados.

Resulta relevante constatar la creciente importancia que adquiere la migración laboral, especialmente en el contexto de un régimen de reproducción demográfica de los países desarrollados, que muestra claras insuficiencias para cubrir la demanda de fuerza de trabajo que requiere la nueva economía. En este contexto, se constata un creciente desequilibrio entre la oferta de puestos de trabajo que genera el crecimiento económico, y que demanda, por tanto, la reproducción del capital y la oferta de mano de obra que la demografía local está en condiciones de generar.

$\mathrm{Al}$ respecto, los datos para Estados Unidos resultan elocuentes. En efecto, entre 2000 y 2012, la economía de ese país generaba, en promedio, empleos 
para 140 millones de personas anualmente. Sin embargo, la demografía de la población nativa no generaba una población económicamente activa inferior a los 120 millones de personas, incluyendo entre ellos a quienes estaban en el desempleo. ${ }^{3}$ Esta situación de déficit permanente y estructural de mano de obra ha debido ser subsanada por el aporte de la inmigración laboral proveniente de países latinoamericanos, principalmente.

Las contribuciones de esta fuerza de trabajo migrante se manifiesta no sólo en la tendencia y composición del mercado de trabajo, sino también, y especialmente, en la misma dinámica de la actividad económica. Entre 2000 y 2012, por ejemplo, los trabajadores migrantes provenientes de países latinoamericanos habrían aportado casi 20\% del crecimiento económico de Estados Unidos, proporción que se eleva a $35 \%$ si se considera el aporte de la población nativa de origen latino. Asimismo, frente a la crisis económica que se desata en 2007, son precisamente estos trabajadores de origen latino los que más han contribuido a mantener a flote la economía estadounidense. En efecto, entre 2007 y 2012, los trabajadores de origen latino aportaron 52\% del crecimiento del producto interno bruto (РIB), más que duplicando el aporte que hicieron en conjunto los trabajadores nativos no latinos. ${ }^{4}$

Estos datos ilustran el nivel de importancia que adquiere la inmigración laboral para sostener el crecimiento económico y, por tanto, para la reproducción ampliada del capital en los países desarrollados. Tenemos claro que la migración no es el único factor, pero también no queda duda que no deja de tener un papel relevante.

En relación con el momento reproductivo, distintos autores han señalado que a través de la migración se activa un sistema de redes sociales y familiares que, sustentadas en principios de solidaridad y reciprocidad, permiten la reproducción de la migración, en lo que se ha denominado proceso de causación acumulativa (Massey, 1990). Tradicionalmente, este proceso de causación acumulativa se ha analizado y entendido como una forma de reproducción de la migración como proceso social, y por tanto de los migran-

${ }^{3}$ Cálculos propios con base en US Census Bureau (2000-2012).

${ }^{4}$ Datos revisados y actualizados, siguiendo el modelo que se propone en Canales (2011a). 
tes, sus familias y comunidades, como principales agentes. En nuestro caso, quisiéramos dar un giro adicional a este mismo mecanismo de reproducción del proceso social de la migración, para focalizarnos en lo que aquí nos interesa, la reproducción de la fuerza de trabajo migrante.

Desde nuestra perspectiva, con la reproducción social de la comunidad y de las familias, no sólo se reproducen las personas así en abstracto, sino que también, y fundamentalmente, se reproduce su posición social en un contexto particular de relaciones económicas y laborales. Si desde la demografía, la antropología o la sociología vemos a las redes sociales y su transnacionalidad como una estrategia de reproducción social de la población, de las familias o de la comunidad, desde la economía, y en particular desde la perspectiva de la reproducción del capital, vemos estos mismos mecanismos como la base social para la reproducción de la fuerza de trabajo migrante en el actual contexto de globalización económica.

Las redes sociales y el capital social de los migrantes y las comunidades son el mecanismo de reproducción de la migración como proceso social. Pero no se trata de la reproducción de una migración en general, así en abstracto, sino de migrantes laborales que se insertan en actividades productivas para el capital en los países de destino participando, de este modo, en las estructuras de diferenciación social, esto es, de clases sociales a nivel de la sociedad global. Si con la migración internacional las comunidades de origen se han transnacionalizado, desde esta otra perspectiva podemos, entonces, decir que también se ha transnacionalizado el momento de la reproducción de la fuerza de trabajo migrante. En el esquema 2 hemos querido ilustrar esta situación, en donde a través de la migración internacional se vincula en un mismo proceso de globalización la reproducción de la fuerza de trabajo migrante con la reproducción del capital.

En este sentido, planteamos una situación paradójica en relación con la migración y la globalización. Aunque los migrantes casi nunca aparecen como uno de los actores que concentre los reflectores de la globalización, su trabajo sí forma parte directa de la globalización del capital, en la medida que constituye, como hemos visto, un elemento sustancial sin el cual la misma dinámica económica de los países desarrollados se vería seriamente mermada.

$22 \frac{\text { SEGUNDO SEMESTRE } 2013}{\text { MIGRACIÓN Y DESARROLLO NÚM. } 21}$ 
Como trabajo, los migrantes participan directamente de la globalización y contribuyen a la reproducción y acumulación del capital a escala global.

Sin embargo, como trabajadores, los migrantes son continua y permanentemente relegados a los confines de la globalización, alejados de todo el glamour que ella encierra, y siempre a la sombra de su trabajo. Siempre como meros actores de reparto, simples extras de una gran producción global. Como trabajadores, su globalización queda restringida a la desterritorialización de los espacios de su reproducción como fuerza de trabajo, esto es, a los espacios restringidos de sus comunidades, que aunque adoptan una forma translocal y transnacional, no necesariamente adoptan una forma global. Su reproducción se podrá haber transnacionalizado, pero en ningún caso se habría mundializado.

\section{ESQUEMA 2}

Migración internacional y reproducción del capital

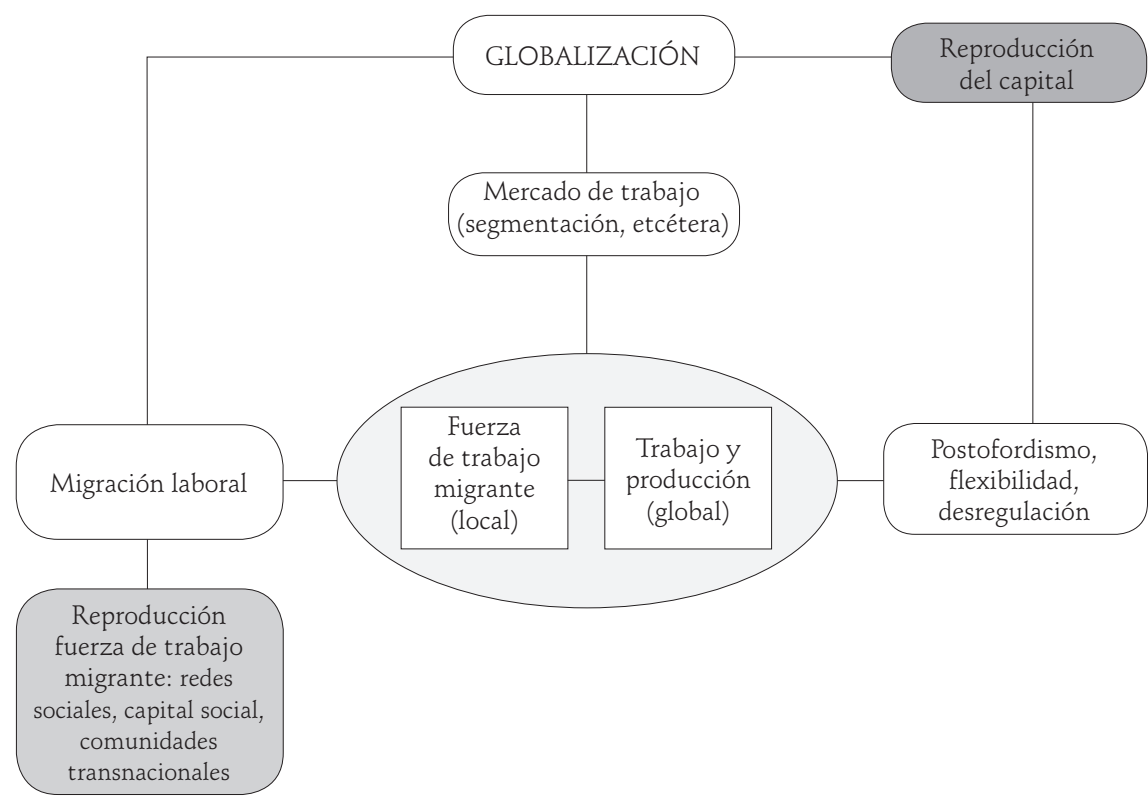

Fuente: Elaboración propia.

En este sentido, la migración configura un mecanismo que articula dos momentos de la globalización económica. Por un lado, como movilidad del 
trabajo contribuye a la globalización desde arriba, esto es, a la globalización del capital y sus actores y representantes. Por otro lado, como movilidad de los trabajadores forma parte de una globalización desde abajo, esto es, contribuye a la desterritorialización de la reproducción de la fuerza de trabajo que requiere el capital global.

El problema para los migrantes es que mientras su trabajo se encuentra materializado en los más diversos productos, artefactos, bienes y mercancías que circulan por todo el mundo, traspasando fronteras y formando parte de la gran farándula de la globalización, ellos, los trabajadores, los que desde abajo son los verdaderos artífices de este proceso, quedan relegados a desplazamientos translocales, a espacios de reproducción localmente situados y restringidos, incluso expuestos a diversas formas de segregación territorial. Sus barrios podrán ser característicos de las ciudades globales, pero no dejan de ser barrios locales, guetos de una globalización que los excluye en su reproducción. He aquí una de las grandes paradojas de la migración: la globalización del trabajo y la producción, esto es, de la reproducción del capital, supone, sin embargo, circunscribir y mantener en ámbitos locales la reproducción de la fuerza de trabajo. Arribamos a una nueva acepción para el término glocalización, que se agrega a las de Bauman (1998) y Robertson (1992): globalización y mundialización del capital y su acumulación, como contracara de la localización de la reproducción de la fuerza de trabajo.

Migración y reproducción social: clases y desigualdad social

A través de la migración se configura un sistema de relaciones entre clases sociales que trasciende y traspasa las fronteras nacionales, y que articula en un mismo proceso las condiciones y dinámicas de la reproducción social en los países de origen con las condiciones y dinámicas de la reproducción social en los países de destino. En particular, vincula el campo y momentos de la reproducción de la fuerza de trabajo migrante (sustentada en el sistema de redes sociales y las comunidades transnacionales) con el momento y campos de la reproducción social de la población de clases medias y altas de las sociedades

$24 \frac{\text { SEGUNDO SEMESTRE } 2013}{\text { MIGRACIÓN Y DESARROLLO NÚM. } 21}$ 
de destino, a través de su creciente participación en los trabajos y ocupaciones propios de la reproducción social.

Migración y redes sociales: la reproducción social de comunidades transnacionales

A través de la migración se activa y expande un complejo sistema de redes y relaciones sociales y familiares más allá de los límites territoriales de la comunidad de origen. A través de este sistema de redes y relaciones sociales la migración deviene mecanismo de reproducción social del migrante, la familia y la comunidad. En efecto, la migración internacional configura una estrategia de sobrevivencia y reproducción social para las familias y las comunidades de origen de la migración (De Has, 2007). De hecho, varios autores ya han señalado cómo a través de las redes sociales y familiares se articulan la inserción laboral en el destino con la reproducción social en el origen. Una vez establecido este sistema de redes sociales transnacionales, él no sólo sirve para sostener el desplazamiento de personas, sino que a través de ese sistema se configura también un continuo flujo de bienes materiales y simbólicos en ambos sentidos, a través del cual se conforma un complejo sistema social cuyas estructuras de relaciones materiales, sociales y simbólicas trascienden las fronteras nacionales y los límites territoriales de las comunidades. Lo relevante es que a través de este sistema de intercambio y circulación de todo tipo se articula la reproducción social de las comunidades de origen con el proceso de reproducción social en los lugares de asentamiento de los migrantes en los países de destino.

Sin embargo, no se trata de la reproducción de la migración y de los migrantes pura y simplemente, sino que a través de ello se da también la reproducción de su posición dentro de la estructura social en los lugares de origen y destino. En este caso, se trata de trabajadores migrantes insertos en condiciones de precariedad laboral, alta flexibilidad, sin prestaciones $y$, en muchas ocasiones, de alta vulnerabilidad social y política derivada de su condición de indocumentados. Las redes sociales, así como las comunidades 
transnacionales, conforman un mecanismo que a la vez que reproduce el proceso social de la migración, también reproduce, en el mismo proceso, la situación social de los migrantes, esto es, su posición relativa frente a los demás estratos y clases sociales.

Así, por ejemplo, a través de las redes sociales los migrantes acceden a empleos y ocupaciones, a viviendas, espacios de vida social, etcétera, pero se trata de campos sociales donde se reproduce su misma situación socioeconómica. Por lo pronto, no siempre acceden al empleo óptimo de acuerdo con sus acreditaciones, experiencia laboral u oficio, sino al empleo que es posible conseguir a través de sus redes sociales (Requena, 1991). En otras palabras, las redes sociales son un capital de gran valor, y de gran poder para enfrentar y lograr la sobrevivencia y reproducción en situaciones sociales adversas, de alta vulnerabilidad, pero no por ello son una estrategia de solución y superación de esas condiciones estructurales que definen y determinan en muchos casos, la posición relativa de los sujetos, en particular su situación de subordinación y de escaso poder de negociación social y política.

Migración y reproducción social en sociedades de destino

En los países centrales, la reproducción social de sus clases medias y altas ha debido adaptarse a estos tiempos de globalización, abriendo espacios para incorporar sujetos sociales (inmigrantes) y campos sociales (sus familias y comunidades) que evidentemente están más allá de los límites de la sociedad y el Estado. Análisis y estudios recientes sobre la inserción laboral de los migrantes documentan su creciente papel en diversas actividades económicas orientadas a la reproducción social y cotidiana de la población nativa de los países de destino, particularmente la de los estratos sociales medios y altos (Vershuur, 2007). Nos referimos a diversas ocupaciones en lo que se ha llamado servicios de proximidad o servicios para la reproducción de la vida cotidiana, que corresponden a aquellas actividades remuneradas que tienen por objeto satisfacer, directamente, necesidades de las personas y las familias, en su ámbito doméstico o bien que conlleven a su reproducción como

$26 \frac{\text { SEGUNDO SEMESTRE } 2013}{\text { MIGRACIÓN Y DESARROLLO NÚM. } 21}$ 
personas y familias. El caso más paradigmático es el del servicio doméstico, pero no es el único. Junto a él, han proliferado muchas otras actividades y ocupaciones orientadas al cuidado de personas enfermas, niños y adultos mayores, preparación de alimentos, limpieza y mantenimiento, entre un largo etcétera.

La mercantilización del servicio doméstico no es algo nuevo en la sociedad capitalista, lo novedoso en la situación actual es que en los países desarrollados la masiva demanda por este tipo de trabajadoras se da en el contexto de cambios sociales y demográficos que ya hemos señalado. De esta forma, el envejecimiento demográfico, la inserción masiva de la mujer al mercado de trabajo, los cambios en la formación y estructura de los hogares, la reducción de la fecundidad, entre otros, impulsan una demanda creciente por trabajadoras que se dediquen a esas labores. Asimismo, las mujeres nativas que antes se dedicaban a estas mismas labores se reducen en volumen, pues crecientemente están optando por otros trabajos menos precarizados y que gozan de mayor valoración y prestigio social y, por ende, de mejores salarios y condiciones de trabajo (Parella, 2003).

Esta situación se manifiesta en un desajuste estructural entre demanda y oferta en este mercado de trabajo, lo que abre el espacio para que mujeres inmigrantes provenientes de países periféricos se incorporen a este tipo de mercados en los países centrales. Los datos para España y Estados Unidos son elocuentes. Para fines de la década pasada, los inmigrantes latinoamericanos aportaban 32\% del servicio doméstico en España y 34\% en Estados Unidos. Asimismo, en este mismo país, los latinoamericanos aportaban la cuarta parte de los trabajadores empleados en servicios de limpieza, mantenimiento, así como de cocineros y preparación de alimentos (Canales, 2011b). Se trata no sólo de trabajos de baja calificación laboral, sino de tareas de muy baja valoración social, lo que redunda en sus bajos salarios, contextos de precariedad, ausencia de marcos regulatorios formales y que encierran un importante grado de desprestigio social. En este contexto, no es de extrañar que surja un proceso de etnoestratificación (Catarino y Oso, 2000) o de racialización de los servicios reproductivos (Hondagneu-Sotelo, 2007), es decir, 
una diferenciación socio-laboral con base en factores étnico-migratorios, más que en las credenciales laborales de cada persona.

Migración y reproducción social: hacia una visión global y comprehensiva

Nuestra tesis es que a través de la migración se configura un sistema global (translocal y transnacional) de reproducción social. En efecto, la migración permite articular el proceso de reproducción social de la población nativa en los lugares de destino con la reproducción social de la población en los lugares y comunidades de origen. El aporte de los migrantes en las ocupaciones y actividades de reproducción social de la población nativa confirman la primera parte de esta tesis. El papel de las redes sociales y familiares así como de las remesas, ambas puestas en operación a partir de la migración misma, dan cuenta de la segunda parte de esta tesis. Se trata, en definitiva, de la articulación de los mecanismos y procesos de reproducción social de clases sociales diferentes y, por ese medio, de la reproducción de un sistema de desigualdad social global y transnacionalizado.

Mientras la migración configura una transferencia de fuerza de trabajo que contribuye a la reproducción de las familias de clases medias y altas en los lugares de destino, los salarios que estas mismas familias le pagan a la migrante, y que ésta envía como remesas a sus familias, constituyen una transferencia en sentido inverso que contribuye a la reproducción de los migrantes y de sus familias, y por ese medio a la reproducción de su fuerza de trabajo, con lo cual se sienta la base para que el circuito de transferencias y flujos de personas, remesas, bienes materiales y simbólicos se renueve una y otra vez. De esta forma, sujetos y poblaciones que aparentemente no tienen ningún vínculo ni contacto directo (las familias de los migrantes en los lugares de origen, por un lado, y la población de clases medias y altas de los lugares de destino, por otro), están vinculadas por la migración laboral, que constituye un mecanismo que, cual bisagra, articula la reproducción de ambas poblaciones tan distintas socialmente y tan distantes territorialmente hablando.

$28 \frac{\text { SEGUNDO SEMESTRE } 2013}{\text { MIGRACIÓN Y DESARROLLO NÚM. } 21}$ 
Nada ejemplifica mejor esta situación que el papel de la migración en la configuración de un sistema de maternidad transnacional, y que puede extenderse también a la llamada transnacionalización de la industria del cuidado (Ehrenteich y Hochschild, 2004). La creciente demanda en los países centrales por mujeres inmigrantes procedentes preferentemente de países del Tercer Mundo para realizar este tipo de tareas de servicio doméstico, cuidado de personas y, en general, de los llamados servicios reproductivos y personales, conlleva una forma emergente de división social del trabajo que adopta una forma transnacional y que se sustenta en una forma de "trasvase de desigualdades de clase y etnia» (Parella, 2003:15), que va desde las mujeres nativas de los países centrales, que se habrían emancipado y liberado de las antiguas cadenas que las ataban a las tareas del hogar, hacia las mujeres inmigrantes que requieren de esos ingresos para su propia reproducción social, y que se ven obligadas a desatender sus propias cargas y responsabilidades reproductivas de sus familias, que se han quedado en sus países de origen. ${ }^{5}$

En estos tiempos de globalización, los procesos de reproducción social -que anteriormente se circunscribían a espacios locales (nacionales)— forman parte de espacios globales (transnacionales). Precisamente, a través de la migración internacional se configuran estos campos globales en donde se produce la intersección entre procesos locales de reproducción social, esto es, espacios que interconectan la reproducción social de unos y otros. El esquema 3 nos permite ilustrar esta tesis sobre la interconexión global de estos espacios locales de reproducción social.

\footnotetext{
${ }_{5}^{5}$ Aunque este no es el momento para entrar en el debate, no podemos pasar por alto la paradoja que implica todo este proceso de emancipación de la mujer en los países centrales. $\mathrm{Al}$ respecto, lo menos que podemos decir, es que se trata de una emancipación fragmentada y que reproduce formas de desigualdad social y de género. Sin duda, favorece en algunos aspectos a las mujeres de los países centrales, pero no rompe necesariamente con la inequidad de género en esos mismos países. Esta desigualdad tan sólo habría sido transferida desde las mujeres nativas hacia las mujeres inmigrantes. En el fondo, la liberación de unas (las mujeres nativas), así sea parcial e incompleta, descansa en cierta forma, en la opresión de otras (las mujeres inmigrantes).
} 


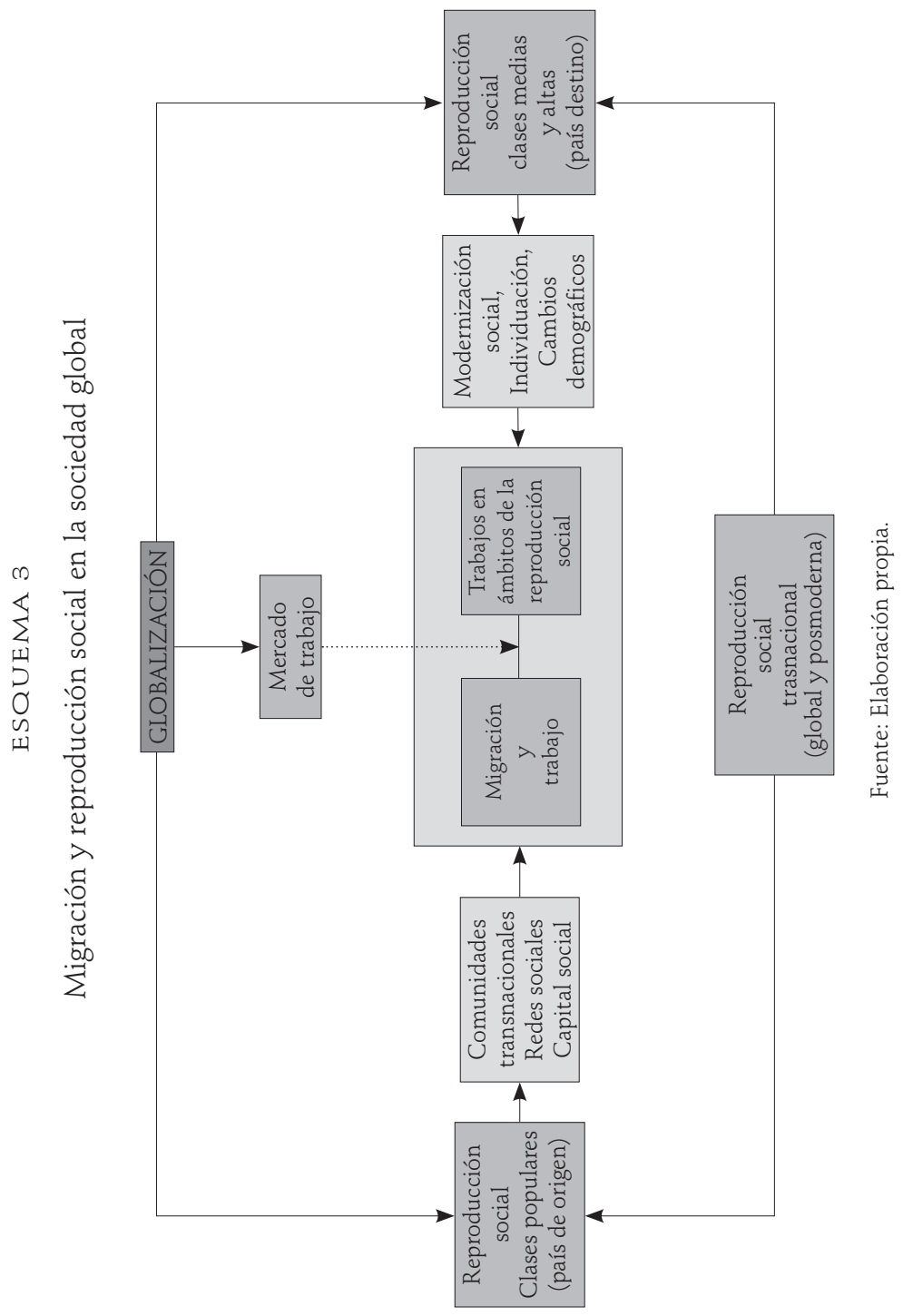


Comúnmente, los estudios sobre la inserción laboral de los migrantes se ha centrado en las condiciones de funcionamiento de los mercados de trabajo en los lugares de destino y, en particular, en cómo a través de la migración se articula una demanda de fuerza de trabajo en los lugares de destino con una oferta de fuerza de trabajo generada en los lugares de origen. En ambos casos, podemos apelar a los enfoques de la globalización, en el sentido que ello nos permite explicar las transformaciones en el funcionamiento de los mercados de trabajo. Nos referimos a los cambios en la estructura de ocupaciones, a la dinámica específica de cada sector de actividad, a los procesos de desregulación contractual y flexibilidad laboral, entre muchos otros (es lo que está representado en la parte central y alta del esquema 3).

En un segundo momento, podemos ampliar este análisis incorporando los cambios sociales y demográficos en las sociedades de destino, que surgen con la misma globalización y advenimiento de una sociedad posmoderna. Nos referimos, por ejemplo, a la creciente incorporación de las mujeres nativas a los mercados de trabajo, a los cambios culturales y de comportamiento social y demográfico vinculados a la posmodernidad, al avance en la segunda transición demográfica y al envejecimiento de las poblaciones de los países desarrollados, entre muchos otros. En este sentido, la inmigración laboral permite, precisamente, llenar no sólo vacíos demográficos que deja el envejecimiento, sino además sustentar estos cambios sociales y culturales de la población nativa (es lo que hemos representado en la parte central-derecha del esquema 3).

Por otro lado, podemos incorporar el papel de las redes sociales y familiares en la conformación de comunidades transnacionales a través de las cuales se genera este mecanismo de causación acumulativa que permite la reproducción social de los migrantes y sus familias a través de la perpetuación de la misma migración internacional. En este sentido, podemos decir que la mirada de la migración se aleja, en parte, de sus aspectos estructurales y contextuales para adentrarse en el proceso social de la migración, esto es, en los espacios de la vida cotidiana y su papel como soportes materiales, sim- 
bólicos y culturales del proceso migratorio (es lo que aparece representado en la parte central-izquierda del esquema 3).

Ahora bien, a lo anterior podemos agregar un tercer nivel de análisis, que no es sino la integración de todo lo anterior, a partir del enfoque de la reproducción social en la sociedad global (es lo que hemos representado en los extremos izquierdo y derecho del esquema 3 ).

- Por un lado, el trabajo de los inmigrantes contribuye a sostener no sólo un modo de vida de la población en los países de destino, sino fundamentalmente a sustentar la reproducción en el marco de las transformaciones sociales, económicas y demográficas que se dan en esta era global y posmoderna. Asimismo, no se trata sólo de la reproducción de la población en abstracto, sino de determinados grupos, estratos y clases sociales $y$, por tanto, de la reproducción de su posición social en una estructura de clases a nivel global.

- Por otro lado, las redes sociales y familiares no sólo contribuyen a la reproducción y perpetuación de la migración, como señalan Massey, Durand y Malone (2009), sino que a través de ello contribuyen a la reproducción y perpetuación de su condición social como comunidades y familias migrantes, esto es, la reproducción de su posición en una estructura social y de clases a nivel global.

Conjuntando estos dos aspectos, podemos afirmar entonces que ellos no son sino las dos caras de un mismo proceso global: la reproducción a nivel global, de una estructura social en la cual podemos identificar diversos estratos, grupos y clases sociales, distintas y distantes, pero articuladas e integradas en su reproducción social. En otras palabras, a través de la migración, la reproducción social de clases medias y bajas en los países de origen está, en cierta medida, articulada con la reproducción social de la población de los estratos medios y altos en los países de destino. Esta integración, o más bien, estos campos de intersección de los procesos locales de reproducción social es lo que queremos destacar en términos de la configuración de un proceso global de reproducción social.

$32 \frac{\text { SEGUNDO SEMESTRE } 2013}{\text { MIGRACIÓN Y DESARROLLO NÚM. } 21}$ 


\section{Conclusiones}

Nuestra tesis es que en la actualidad la migración internacional es un fenómeno que desde diversos ámbitos y desde diversos niveles contribuye a la estructuración de la sociedad contemporánea. Para entender este proceso de estructuración, proponemos analizar la migración desde un enfoque de la reproducción, esto es, analizar la migración como un factor de la reproducción de la sociedad y, por ese medio, de su transformación. Sin duda, el modelo de la reproducción configura un gran puzzle, un rompecabezas con infinidad de piezas, muchas de las cuales, además, se superponen y engarzan de más de una manera. En nuestro caso, hemos optado por analizar sólo tres de ellos, que corresponden a la migración como factor de reproducción de las poblaciones, de reproducción del capital y de reproducción de las estructuras de clases y de desigualdad social. Cada uno de estos niveles o campos de la reproducción, por sí mismo incluyen un amplio bagaje teórico y metodológico que ha derivado en muchas investigaciones y hallazgos particulares.

Asimismo, mucho de lo que hemos expuesto en este artículo no hace sino retomar lo que muchos autores han dicho antes, y en mejor forma. Nuestro aporte, en tal sentido, está más bien en darles a ellos una mirada de conjunto, derivada de una imagen general que tenemos de este gran rompecabezas, y desde la cual derivamos la posición, función y contradicciones que corresponden a cada una de estos tres grandes momentos de la reproducción de la sociedad: demografía, economía y clases sociales. En este sentido, lo que proponemos es traspasar los tradicionales límites de cada nivel de análisis de la migración para configurar un nivel de segundo orden que se construye a partir de la integración de los tres anteriores. Se trata de trascender el significado de la migración en cada nivel para arribar a un nuevo nivel de comprensión de la migración.

- Desde la demografía podemos entender cómo esta reproducción translocal de la migración es también una reproducción translocal de las poblaciones, conformando un sistema global de reproducción demográfica. Se trata de la 
articulación de patrones de reproducción demográfica aparentemente independientes, pero que a través de la migración se integran en un esquema de complementariedad demográfica. En un caso, la migración constituye una válvula de escape ante un bono demográfico que la economía local no es capaz de hacer efectivo. Por otro lado, la misma migración permite llenar los vacíos demográficos que deja el envejecimiento de la población y la reducción de la fecundidad ante el avance de la segunda transición demográfica.

- Desde la economía, podemos además entender el sentido de esta reproducción demográfica, al menos desde tres ópticas complementarias. Por un lado, el análisis de la globalización económica nos indica que no se trata de la migración de personas pura y simplemente, sino de una migración que es esencialmente laboral, y por ese medio, un mecanismo para proveer fuerza de trabajo para el capital. Asimismo, no es tampoco la migración de un trabajador pura y simplemente, sino la de un grupo de trabajadores socialmente vulnerables e insertos en trabajos precarios, flexibles e inestables. Por último, las remesas que envían los migrantes no son sino la forma de transferir el salario para asegurar la reproducción de sus familias, cerrando de ese modo el círculo de la reproducción del capital al sustentar la articulación del momento reproductivo local (de la fuerza de trabajo, a través de sus comunidades y familias de origen) con los momentos productivos globales (trabajo e inserción laboral en economías de destino).

- Por último, esta reproducción de las poblaciones de origen y destino así como del capital, es también la reproducción de diversos sujetos sociales, esto es, de personas insertas en diferentes posiciones dentro de la estructura social. Por un lado, a través de la inserción en ocupaciones del ámbito de la reproducción social en los lugares de destino, los migrantes contribuyen a sustentar la reproducción de la población de clases medias y altas en esas sociedades, posibilitando su reproducción material, así como de un estilo de vida y un patrón de comportamiento social (individuación, emancipación de la mujer, calidad de vida, entre otros aspectos). Por otro lado, la misma migración y las remesas conforman una estrategia de reproducción de los mismos migrantes, sus familias y sus comunidades. La migración configura así una especie de bisagra que vincula la reproducción de sujetos distintos socialmente y distantes geográficamente en lo que podemos denominar como la 
configuración de un sistema global y transnacional de reproducción de la estructura de clases y desigualdad social propio de la sociedad contemporánea.

La mirada desde estos tres niveles nos permite entender que la migración no es sólo un fenómeno de desplazamiento de personas, sino que a través de ella se configura un complejo sistema de complementariedades y transferencias de todo tipo, las cuales involucran a personas en determinadas posiciones sociales, económicas y laborales. A la vez que forma parte de un sistema global de reproducción demográfica, a través de ella también se posibilita la reproducción del sistema económico y social, al menos en lo que se refiere a la polarización y segmentación de los mercados de trabajo y, por ese medio, de las formas de desigualdad social en esta era de globalización.

En síntesis, esta visión integrada de estas tres dimensiones o momentos de la migración internacional (demografía, economía, clases sociales) nos permite alcanzar un nuevo nivel de comprensión de la migración internacional, como componente y artífice de la reproducción de la sociedad global contemporánea. En el esquema 4 intentamos ilustrar esta idea del papel de la migración en la reproducción de la sociedad, a través de su función en la reproducción del capital, de la población y de la estructura de clases y desigualdad social.

Este análisis desde la perspectiva de la reproducción de la sociedad nos permite leer e interpretar la migración internacional con base en otros significados sociales, económicos, demográficos y culturales. Si, por un lado, la migración es un factor que contribuye a la reproducción de la sociedad contemporánea, por otro lado, y con base en esa misma contribución, la migración también constituye un factor que contribuye a la transformación de esa misma sociedad. No es que la migración se constituya en el único o más importante factor de cambio de esta sociedad, pero, sin duda, es un fenómeno social que también participa de los dilemas y contradicciones de la sociedad actual, que conllevan a su necesario movimiento y transformación.

La sociedad contemporánea enfrenta diversos dilemas y tensiones. Uno de ellos, que es de particular interés para nuestra discusión, es el reto que 


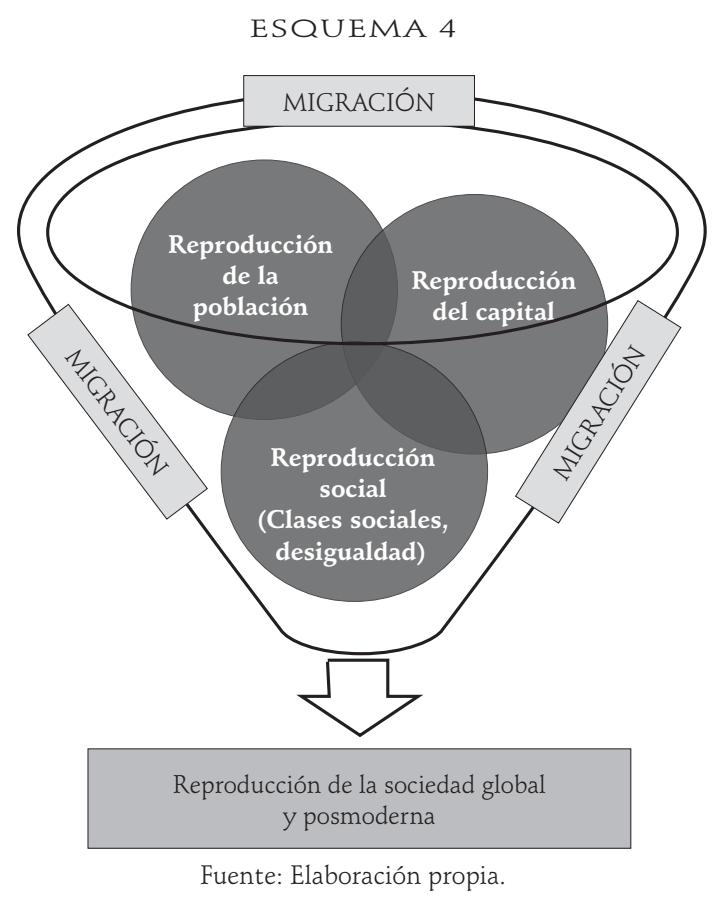

plantea el cambio demográfico actual en las sociedades avanzadas. Se trata de sociedades con bajas tasas de fecundidad y en proceso envejecimiento demográfico. Como hemos visto, se trata de un cambio que afecta directamente la capacidad de la demografía local de generar los contingentes de población y de fuerza de trabajo necesarios para sustentar el crecimiento económico y, por ese medio, la reproducción y acumulación de capital, así como la reproducción social de la población nativa.

En este contexto demográfico, la inmigración de trabajadores, especialmente provenientes de países periféricos, constituye una opción atractiva para esas sociedades avanzadas. Por un lado, proveen la población necesaria para mantener los niveles de reproducción demográfica y, por otro lado, proveen la fuerza de trabajo necesaria, tanto para actividades directamente productivas (construcción, industria tradicional, etcétera) como en ocupaciones de la reproducción (servicio doméstico, industria del cuidado, preparación de alimentos, limpieza y mantenimiento, etcétera). No sólo eso, sino que además 
proveen mano de obra relativamente barata, en la medida que parte de su reproducción económica se sustenta en las mismas redes sociales y familiares que tejen esos migrantes entre sus comunidades de origen y los asentamientos en los lugares de destino.

Sin embargo, en esos mismos procesos se encierra un dilema no menor. Por un lado, al sustentar la reproducción de los migrantes en esas mismas redes sociales, hace que ellas adopten una gran fortaleza, reproduciendo no sólo a la población y la fuerza de trabajo, sino también, especialmente, reproduciendo estilos de vida, sistemas de valores, patrones de comportamiento, identidades colectivas, entre otros aspectos que mantienen y reproducen una importante distancia social y cultural respecto a las posiciones hegemónicas y dominantes de las sociedades de destino. Lejos de asimilarse a la cultura dominante, configuran formas culturales, sociales y simbólicas alternativas, que no hacen sino reforzar en los países de destino su condición de sociedades multiculturales, con creciente diferenciación étnica y desigualdad social.

Por otro lado, no se trata de minorías demográficas, que en otros contextos y momentos, muy bien pudieran haberse asumido y tolerado como desviaciones aceptables de la cultura y patrones sociales dominantes y hegemónicos. Por el contrario, se trata de grupos demográficos en continuo crecimiento que, en algunos casos, incluso pueden llegar a cuestionar la tradicional primacía de los grupos demográficos nativos.

Es el caso de Estados Unidos, por ejemplo, en donde ya hoy en día los hispanos no sólo son la principal minoría étnica, sino además la de mayor crecimiento demográfico. Asimismo, su comportamiento demográfico es totalmente diferente de la población blanca nativa. Mientras estos últimos están avanzando en la segunda transición demográfica, con tasas de fecundidad incluso por debajo de los niveles de reproducción, la población hispana mantiene altos niveles de reproducción demográfica, de tal forma que, hoy en día, prácticamente un cuarto del total de nacimientos en ese país es un bebé cuya madre es de origen hispano. Este comportamiento reproductivo, junto a la masividad de la inmigración, lleva a estimar que en un futuro no muy lejano, la población blanca nativa deje de ser una mayoría absoluta, 
para representar menos de $50 \%$ de la población. Asimismo, se estima que para la década de los cuarenta, la población de origen hispano represente casi un tercio del total de la población de Estados Unidos.

Son insospechadas las consecuencias que tendría un cambio de tal magnitud en la composición étnica de la población de Estados Unidos. Se trata, en el fondo, del cuestionamiento de la hegemonía social, política y cultural de la actual población blanca en ese país. Sin embargo, el problema para Estados Unidos, en este caso, es que por ahora no tiene alternativas viables para enfrentar este dilema. Cerrarse a la inmigración pone en peligro no sólo la reproducción social de sus clases medias y altas, sino también pone en riesgo su propio crecimiento económico, y por ese medio pone en riesgo su fortaleza como potencia económica mundial. La reproducción del capital requiere de proveerse de esa mano de obra para mantener así sus hegemonías a nivel local e internacional. Sin embargo, la única opción viable por el momento es proveer esa fuerza de trabajo a través de la inmigración masiva desde países periféricos, con la consecuente transformación en la composición étnico-demográfica de su población, lo cual, sin embargo, pone en riesgo los actuales equilibrios demográficos sobre los cuales se sustenta, en parte al menos, la distribución del poder en esa misma sociedad.

Para el capital, el dilema pareciera ser «transformación demográfica o desaparecer». El problema es que para la población nativa blanca de Estados Unidos esa transformación demográfica es una forma, si no de desaparecer, sí de reducir sus ámbitos de poder, influencia social, cultural y, sobre todo, política a nivel no sólo local, sino, principalmente, a nivel mundial.

\section{Bibliografía}

Bauman, Zygmunt (1998), "On Glocalization: or Globalization for Some, Localization for Some Others», Thesis Eleven, vol. 54, núm. 1.

BeCK, Ulrich (2000), Un nuevo mundo feliz. La precariedad del trabajo en la era de la globalización, Barcelona, Paidós.

$38 \frac{\text { SEGUNDO SEMESTRE } 2013}{\text { MIGRACIÓN Y DESARROLLO NÚM. } 21}$ 
(1998), Qué es la globalizacion. Falacias del globalismo, respuestas a la globalización, México, Paidós. blications.

Canales, Alejandro I. (2011a), "Las profundas contribuciones de la migración latinoamericana a los Estados Unidos», en Jorge Martínez Pizarro (ed.), Migración internacional en América Latina y el Caribe. Nuevas tendencias, nuevos enfoques, Santiago, CEPAL.

(2011b), «Latin America in the recent wave of international migration», en José Antonio Ocampo y Jaime Ros (eds.), The Oxford Handbook of Latin American Economics, Oxford University.

CAstles, Stephen y Mark J. Miller (1993), The Age of migration. International Population Movements in the Modern World, Nueva York, Guilford Press.

CASTRo, Antonio Barros de y Carlos F. Lessa (1991) [1969], Introducción a la economía: un enfoque estructuralista, México, Siglo XXI.

Catarino, Christine y Laura Oso (2000), «La inmigración femenina en Madrid y Lisboa: hacia una etnización del servicio doméstico y de las empresas de limpieza», PAPERS, Revista de Sociología, núm. 60, Universidad Autónoma de Barcelona, pp. 183-207.

Cooke, Martin (2003), «Population and Labour Force Ageing in Six Countries», Workforce Aging in the New Economy, Working Paper (4).

De Has, Hein (2007), «Remittances, Migration and Social Development. A Conceptual Review of the Literature», United Nations, Research Institute for Social Development, Social Policy and Development Programme, Paper Number 34. Ehrenteich, Barbara y Arlie Russel Hochschild (2004), Global Woman. Nannies, Maids and Sex Workers in the New Economy, Nueva York, Henry Holt and Company.

Giddens, Anthony (1984), The Constitution of Society, Cambridge, Polity Press.

GuzMán, José Miguel (2002), «Envejecimiento y desarrollo en América Latina y el Caribe», Serie Población y Desarrollo, 28, Santiago de Chile, Centro Latinoamericano y Caribeño de Demografía (Celade)-División de Población.

Herrera Ponce, María Soledad (2007), Individualización social y cambios demográficos: ihacia una segunda transición demográfica?, Centro de Investigaciones Sociológicas, Colección monografías, núm. 232, Madrid, España.

Hondagneu-Sotelo, Pierrete (2007), Doméstica: Immigrant Workers Cleaning and caring in the Shadows of Affluence, Los Ángeles, University of California Press. 
INE (2013), Indicadores demográficos básicos, España, Instituto Nacional de Estadística, http://www.ine.es/jaxi/menu.do? $\mathrm{L}=0 \&$ divi $=I D B \&$ his $=0 \&$ type $=\mathrm{db}$ (consultado el 28 de febrero de 2013).

Lipietz, Alain (1997), El mundo del post-fordismo, Jalisco, México, Cuadernos del Cusch, Universidad de Guadalajara, en: http://www.revistas.unal.edu.co/index.php/ede/article/viewFile/23729/24410.

MASSEY, Douglas (1990), «Social Structure, Household Strategies, and Cumulative Causation of Migration», Population Index, vol. 56, núm. 1. , Jorge Durand y Nolan Malone (2009), Detrás de la trama. Políticas migratorias entre México y Estados Unidos, México, Miguel Ángel Porrúa, Universidad Autónoma de Zacatecas.

Maturana, Humberto (2009), La realidad: iobjetiva o construida? I Fundamentos biológicos de la realidad, Barcelona, Antrophos/Universidad Iberoamericana/ Instituto Tecnológico de Estudios Superiores de Occidente.

Organización de las Naciones Unidas (ONU) (2001), Replacement Migration: Is It a Solution to Declining and Ageing Populations?, United Nations, Population Division. United Nations Publication, ST/ESA/SER.A/206.

Parella Rubio, Sonia (2003), Mujer, inmigrante y trabajadora: la triple discriminación, España, Editorial Anthropos.

Redondo, Nélida y Sagrario Garay (2012), El envejecimiento en América Latina. Evidencia empírica y cuestiones metodológicas, Asociación Latinoamericana de Población, Serie Investigaciones 13, Río de Janeiro, Brasil.

Requena S., Felix (1991), Redes sociales y mercado de trabajo. Elementos para una teoría del capital relacional, Madrid, Centro de Investigaciones Sociológicas.

Robertson, Roland (1992), Globalization. Social Theory and Global Culture, Londres, Sage Publications.

SASSEn, Saskia (2007), Una sociología de la globalización, Buenos Aires, Katz Editores.

US Census Bureau (2000-2012), Current Population Survey, march supplement.

US Department of Health and Human Services (2010), Births: Final Data for 2008. National Vital Statistics Report, vol. 59, núm. 1, diciembre, U.S. Department of Health and Human Services, Centers for Disease Control and Prevention, National Center for Health Statistics, National Vital Statistic System.

VAN DE KAA, Dirk (1987), «Europe’s second demographic transition», Population Bulletin, vol. 42, núm. 1.

$40 \frac{\text { SEGUNDO SEMESTRE } 2013}{\text { MIGRACIÓN Y DESARROLLO NÚM. } 21}$ 
VershUUR, Christine (2007), «Inmigrantes y nueva división internacional del trabajo y de los cuidados», en Isabel Yépes del Castillo y Gioconda Herrera (eds.), Nuevas migraciones latinoamericanas a Europa. Balances y desafíos, Ecuador, Flacso, Obreal, GRIAL y Universidad de Barcelona.

Wimmer, Andreas y Nina Glick-Schiller (2002), «Methodological nationalism and beyond: nation-state building, migration and the social sciences», Global Networks. A Journal of Transnational Affairs, vol. 2, núm. 4. 
